\title{
Skin Toxicity Assessment of Silver Nanoparticles in a 3D Epidermal Model Compared to 2D Keratinocytes
}

This article was published in the following Dove Press journal: International Journal of Nanomedicine

\begin{abstract}
Liang Chen, ${ }^{1, *}$ Meiyu $\mathrm{Wu}^{2, *}$ Shan Jiang, ${ }^{1,3, *}$ Yanyun Zhang, ${ }^{4}$ Runzhi Li, ${ }^{4}$ Yongbo Lu, ${ }^{4}$ Lin Liu, (D) $)^{2}$ Gang Wu, (D) ${ }^{3}$ Ying Liu, $\mathbb{D}^{5}$ Liming Xie, ${ }^{2}$ Liming $X u^{\prime}$

'NCNST-NIFDC Joint Laboratory for Measurement and Evaluation of Nanomaterials in Medical Applications, National Institutes for Food and Drug Control, Beijing 102629, People's Republic of China; ${ }^{2}$ CAS Key Laboratory of Standardization and Measurement for Nanotechnology, NCNST-NIFDC Joint Laboratory for Measurement and Evaluation of Nanomaterials in Medical Applications, Center for Excellence in Nanoscience, National Center for Nanoscience Nanoscience, National Center for Nanoscience and Technology, Beijing 100190, People's Republic of China; ${ }^{3}$ Department of Preclinical Medicine and Forensic, Baotou Medical College, Baotou 014040 People's Republic of China; ${ }^{4}$ Guangdong Biocell Biotechnology Co. Ltd, Dongguan, 523808, Guangdong, People's Republic of China; ${ }^{5}$ CAS Key Laboratory for Biomedical Effects of Nanomaterials and Nanosafety, NCNST-NIFDC Joint Laboratory for Measurement and Evaluation of Nanomaterials in Medical Applications, Center of Nanomaterials in Medical Applications, Center Nanoscience and Technology, Beijing 100190 , People's Republic of China

*These authors contributed equally to this work
\end{abstract}

Correspondence: Liming $\mathrm{Xu}$ NCNST-NIFDC Joint Laboratory for Measurement and Evaluation of Nanomaterials in Medical Applications, National Institutes for Food and Drug Control, Beijing, People's Republic of

China

Email xuliming@nifdc.org.cn

Liming Xie

CAS Key Laboratory of Standardization and Measurement for Nanotechnology, NCNST-NIFDC Joint Laboratory for

Measurement and Evaluation of Nanomaterials in Medical Applications, Center for Excellence in Nanoscience, National Center for Nanoscience and Technology, Beijing, People's Republic of China

Email xielm@nanoctr.cn
Introduction: Increased use of silver nanoparticles (AgNPs) has raised concerns that AgNPs may induce toxic effects. In vitro studies of cell monolayers and in vivo studies have produced conflicting results. The inconsistency of these results has been mainly due to limitations of two-dimensional (2D) monolayer cell systems.

Methods: A three-dimensional (3D) epidermal model called EpiKutis ${ }^{\circledR}$, which exhibits good tissue viability and barrier function was developed. The cytotoxicity of AgNPs against EpiKutis was compared to that against 2D keratinocytes at equivalent AgNPs doses $(0.035,0.07,0.14$, 0.28 , and $0.56 \mathrm{ng}$ per cell). The amount and distribution of AgNPs in the 3D EpiKutis and 2D keratinocytes after exposure were determined. The toxic mechanisms of AgNPs, such as oxidative stress and production of pro-inflammatory cytokines, were investigated.

Results: The results demonstrated that cell viability was greater than $80 \%$ and lactate dehydrogenase (LDH) release did not increase even at the highest dose of AgNPs in EpiKutis. In contrast, treatment of 2D keratinocytes with AgNPs resulted in dose-dependent decrease in cell viability from $63 \%$ to $11 \%$, and a dose-dependent increase in $\mathrm{LDH}$ release from $8 \%$ to $16 \%$. Cytotoxicity of AgNPs in 2D keratinocytes was related to oxidative damage and inflammation, as evidenced by increased levels of reactive oxygen species (ROS), malondialdehyde (MDA), IL-1 $\alpha$, IL-6, and IL-8. In addition, levels of superoxide dismutase (SOD) were decreased. EpiKutis treated with AgNPs did not exhibit increased oxidative damage or inflammation, which may have been due to the barrier properties of the $3 \mathrm{D}$ structure, resulting in reduced penetration of AgNPs. At equivalent per cell doses, total silver penetration into EpiKutis was 0.9 $\pm 0.1 \%$, and total silver penetration into $2 \mathrm{D}$ keratinocytes was $8.8 \pm 0.6 \%$ detected by ICP-MS. The penetration and distribution of AgNPs in 2D keratinocytes were confirmed by the TEM-EDS analysis, which was not found in the 3D EpiKutis. These results showed that AgNPs penetrated EpiKutis to a lesser degree than they penetrated 2D keratinocytes, which suggested that EpiKutis exhibited significant barrier function.

Discussion: The results of this study showed that AgNP toxicity should be evaluated using $3 \mathrm{D}$ epidermal models, which may provide better estimates of in vivo conditions than $2 \mathrm{D}$ models. The EpiKutis model may be an ideal model for assessment of nanotoxicity.

Keywords: silver nanoparticles, AgNPs, cytotoxicity, keratinocytes, 3D epidermal model, EpiKutis $^{\circledR}$, oxidative stress, inflammatory response

\section{Introduction}

Increased use of nanotechnology for manufacture of consumer products has led to concerns regarding safety risks associated with exposure to nanomaterials. Silver nanoparticles (AgNPs) are one of the most widely used nanomaterials due to their 
excellent broad-spectrum antibacterial properties. Silver nanoparticles are present in many industrial and medical products including textiles, food packing, water disinfectants, implant coatings, catheter coatings, and wound dressings. $^{1-3}$ Silver nanoparticles have been reported as ingredients in $30 \%$ of products that contain nanomaterials. ${ }^{4}$ However, AgNPs may be toxic. Therefore, it is imperative to accurately assess potential hazards associated with administration of AgNPs.

Toxicity of AgNPs has been studied extensively over recent decades. Most studies used two-dimensional (2D) cell culture models or animal models to evaluate toxicity. However, few studies have used three-dimensional (3D) tissue models. ${ }^{5-8}$ Use of 2D monolayer cultures comprised of immortalized cell lines, stem cells, or primary cells is the most common method for evaluation of toxicity in vitro. Cell type depends on the proposed application of the nanomaterials and the expected in vivo target organs. ${ }^{9}$ Two-dimensional cell culture is easy to use for biochemical assays. ${ }^{10}$ However, 2D cultures lack cell-cell and cell-matrix interactions and do not exhibit barrier functions. Therefore, common 2D cell culture models generally fail to mimic the in vivo skin microenvironment, and therefore provide limited information regarding physiological responses of organisms to external stimuli, such as nanoparticles. ${ }^{11,12}$

Studies have shown that in vitro and in vivo data often correlate poorly. ${ }^{13}$ As such, care should be taken when generalizing in vitro outcomes to in vivo effects. In recent years, some new drugs have been withdrawn during preclinical studies because in vitro toxicity testing failed to identify hazards associated with these drugs. ${ }^{14}$ In vivo studies are typically performed using a range of doses based on results of in vitro experiments or at actual exposure doses, ${ }^{15}$ which may produce more reliable results. However, use of animals can be a limiting factor in determination of toxicity due to cost, biological safety, and animal ethics. To narrow the in vitro-in vivo gap, there is a strong demand for novel in vitro model systems that can be used to accurately predict in vivo toxicity. ${ }^{16,17}$ An ideal model would allow for in vitro evaluation of the adverse effects of actual doses on, for example, inflammation, reactive oxygen species (ROS) generation, and activation of the immune system, to accurately assess potential hazards associated with nanoparticles.

Cells in 3D tissue models, unlike cells in 2D models, can differentiate and develop cell subsets with different functional states and can form tissue structures. Three-dimensional tissue models can also simulate barrier function to mimic absorption and distribution of materials in vivo. ${ }^{18}$ Therefore, $3 \mathrm{D}$ models may be able to bridge the gap between in vitro and in vivo models. ${ }^{19,20}$ In vitro studies of the biological effects of nanoparticles using 3D model systems may be more appropriate than using 2D model systems because toxicity can be influenced by the cellular microenvironment. ${ }^{21,22}$ Lee et al evaluated the toxicity of nanoparticles using a 3D spheroid culture-based testing system for the first time in $2009 .^{23}$ They found that the toxic effects of CdTe and Au nanoparticles were significantly reduced in spheroid cell culture compared to those in 2D cell culture. In addition, several different in vitro 3D models have been established and used to assess biological effects of nanoparticles. These systems include 3D cell spheroid culture systems ${ }^{24-26}$ and the EpiDerm ${ }^{\mathrm{TM}}$ tissue model. ${ }^{27}$

Rapid development of wearable textiles and medical devices that contain AgNPs has raised concern that consumers may be exposed to increased risk of adverse health effects through dermal exposure to AgNPs. ${ }^{28,29}$ Previous studies showed that AgNPs induced severe cytotoxicity in cultured keratinocytes, but in vivo studies showed relatively weak toxicity. ${ }^{30}$ A previous report showed that no significant toxicological changes occurred during a 28-day AgNP inhalation study in rats. ${ }^{31}$ In the present study, a 3D epidermal model called EpiKutis ${ }^{\circledR}$, comprised of human keratinocytes (KC), was developed to mimic the human epidermis and used to evaluate AgNP toxicity. Twodimensional keratinocyte cultures were used as controls. Oxidative damage and inflammation were assessed in each model.

\section{Materials}

Keratinocytes derived from human foreskin. The collection of human foreskin was approved by the local ethics committee (the fourth people's hospital of Shanxi province, China). The tissue culture media, including tissue under-liquid (TU), tissue air-liquid 1 (TA1), and tissue air-liquid 2 (TA2) were purchased from Guangdong Biocell Biotechnology Co. Ltd (Dongguan Guangdong). 3-(4,5-Dimethyl-2-thiazolyl)-2,5-diphenyl-2H-tetrazolium bromide (MTT) and Triton X-100 were purchased from Sigma (Beijing, China). N-Acetylcysteine (NAC) was purchased from Vetec (Shanghai, China). Lactate dehydrogenase (LDH) cytotoxicity assay kit and enhanced BCA protein assay kit were purchased from Beyotime (Shanghai, China). Acetaminophen was purchased from Solarbio (Beijing, China). ELISA kits for interleukin-1 alpha (IL-1 $\alpha$ ), interleukin-6 (IL-6), interleukin-8 (IL-8), 
and tumor necrosis factor-alpha (TNF- $\alpha$ ) were purchased from R\&D (Shanghai, China). Reactive oxygen species (ROS) detection reagents were purchased from Invitrogen (Paisley PA4 9RF, UK). Total superoxide dismutase (T-SOD) assay kit (hydroxylamine method) and microscale malondialdehyde (MDA) assay kit (TBA method) were purchased from Jiancheng Bioengineering Institute (Nanjing, China). Silver nanoparticles (AgNPs) were purchased from Nanux Inc. (Seoul, Korea).

\section{Methods}

\section{Construction and Histological Characterization of the EpiKutis Model}

Keratinocytes $\left(5 \times 10^{5}\right)$ were seeded on the permeable membranes of transwell chambers, which were then placed in 24well plates and cultured in TU medium at $37^{\circ} \mathrm{C}$ in a $5 \% \mathrm{CO}_{2}$ atmosphere. After $24 \mathrm{hrs}$, the TU medium was discarded and replaced with $200 \mu \mathrm{L}$ of fresh medium. After an additional 24 hrs, the TU medium was exchanged for TA1 medium, and keratinocytes were cultured at the air-liquid interface. The TA1 medium was changed once per day. After culturing for 4 days, the TA1 medium was replaced with TA2 medium, and the cells were cultured for additional 8 days, with daily medium replacement. The result of this procedure was a complete EpiKutis 3D model on the permeable membrane of the transwell chamber.

Harvested EpiKutis was fixed in 4\% paraformaldehyde. The samples were then dehydrated, embedded in paraffin, and cut into 5- $\mu \mathrm{m}$ sections. After hematoxylin and eosin staining, the sections were histologically evaluated.

\section{Assessment of Cell Viability and Barrier Function of the EpiKutis Model}

To assess the cell viability of the model, $300 \mu \mathrm{L}$ of MTT solution $(1 \mathrm{mg} / \mathrm{mL})$ was added to each well. After $3 \mathrm{hrs}$ of incubation at $37^{\circ} \mathrm{C}$, the MTT solution was discarded and each well was rinsed 3 times with PBS. Two milliliters of isopropanol were added to each well, and the 24-well plates were sealed and kept at $4^{\circ} \mathrm{C}$ overnight. After the model systems were thoroughly dissolved, $200 \mu \mathrm{L}$ of lysate was collected and transferred to a 96-well plate (at least two duplicate wells were prepared per sample), and the absorbance at $570 \mathrm{~nm}$ was measured. In each batch of models, cell viability was determined in triplicate at each time point.

The normal human epidermis depends on the stratum corneum as a barrier structure to resist the penetration of foreign chemicals. The barrier function of the EpiKutis model was characterized by evaluating the penetration of $1 \%(\mathrm{v} / \mathrm{v})$ Triton X-100. In each batch of EpiKutis models, cell viability was evaluated in triplicate using the MTT assay prior to the addition of $1 \%$ Triton $\mathrm{X}-100$. Then, $80 \mu \mathrm{L}$ of Triton X-100 was added to the surfaces of other model preparations in the same batch. After either $2 \mathrm{hrs}$ or $4 \mathrm{hrs}$ of exposure, the models were rinsed with PBS, and cell viability was evaluated using the MTT assay. The Triton X-100 exposure time (ET) required to reduce cell viability by $50 \%\left(\mathrm{ET}_{50}\right)$ was used as an index of the barrier function of the EpiKutis model. To verify the stability of the model, barrier function was evaluated in multiple EpiKutis batches.

\section{Characterization of Silver Nanoparticles}

Transmission electron microscopy (TEM, HT7700, Hitachi, Japan) was used to image the morphology of AgNPs. Samples were prepared by placing $6 \mu \mathrm{L}$ of AgNP suspension on a copper grid and allowing the drops to air dry. The AgNPs were then visualized using an accelerating voltage of $80 \mathrm{kV}$. The UV/vis absorption spectra of the AgNPs were recorded using a SpectraMax ${ }^{\circledR}$ i3 microplate reader (Molecular Devices, Shanghai, China) from $200 \mathrm{~nm}$ to 800 nm. Zeta potential and hydrodynamic diameter of AgNPs (20 $\mu \mathrm{g} / \mathrm{mL}$ in deionization water) were measured using a Zetasizer Nano ZS (Malvern, UK). The chemical composition of the nanoparticles was characterized by X-ray photoelectron spectroscopy (XPS, ESCALAB250Xi, Thermo Scientific, USA).

\section{Determination of Equivalent Doses of AgNP Exposure}

The number of cells in 2D cell culture is much lower than that in $3 \mathrm{D}$ cell culture when the cells are confined to the same surface area. Therefore, we normalized the dose of AgNPs to the number of cells to provide doses equivalent to the $2 \mathrm{D}$ and $3 \mathrm{D}$ models.

The EpiKutis model contained about $3.6 \times 10^{5}$ cells per well, as determined using a hemocytometer following trypsinization. The number of cells in the $2 \mathrm{D}$ cultures was approximately $1 \times 10^{4}$ cells per well. These results suggested that the 3D model should be exposed to 36times more AgNPs than the 2D model to achieve equivalent doses.

The doses of AgNPs used for the 3D model were 200 $\mu \mathrm{L}$ of $62.5,125,250,500$, or $1000 \mu \mathrm{g} / \mathrm{mL}$. The doses of 
AgNPs used for the 2D model were $200 \mu \mathrm{L}$ of $1.75,3.5,7$, 14 , or $28 \mu \mathrm{g} / \mathrm{mL}$. The equivalent AgNP doses were 0.035 , $0.07,0.14,0.28$, and $0.56 \mathrm{ng}$ per cell.

\section{Toxicity of AgNPs}

The MTT and LDH assays were used to assess the cytotoxicity of AgNPs. Briefly, $200 \mu \mathrm{L}$ of AgNPs were added to the EpiKutis model surface $(62.5-1000 \mu \mathrm{g} / \mathrm{mL})$ or to the surface of the 2D keratinocytes $(1.75-28 \mu \mathrm{g} / \mathrm{mL})$, each of which had been cultured for $24 \mathrm{hrs}$ after seeding. One percent Triton X-100 and PBS were used as positive and negative controls for the $\mathrm{LDH}$ assay, respectively. After $24 \mathrm{hrs}$ of incubation at $37^{\circ} \mathrm{C}, 50 \mu \mathrm{L}$ of medium from each model preparation was transferred to a 96-well plate for quantitation of $\mathrm{LDH}$ at $490 \mathrm{~nm}$. The formula for determination of $\mathrm{LDH}$ release ratio was as follows:

$\mathrm{LDH}$ release ratio $(\%)=(\mathrm{T}-\mathrm{N}) /(\mathrm{P}-\mathrm{N}) \times 100 \%$

In this formula, $\mathrm{P}, \mathrm{N}$, and $\mathrm{T}$ represented absorption values of the positive control, negative control, and AgNPtreated groups, respectively.

The model systems were washed with PBS, and the cell viability of the $3 \mathrm{D}$ model was assessed using the MTT assay. For 2D keratinocytes, $250 \mu \mathrm{L}$ of MTT $(1 \mathrm{mg} / \mathrm{mL})$ diluted with fresh culture medium was added to each well. After $4 \mathrm{hrs}$ of incubation at $37^{\circ} \mathrm{C}$, the supernatant was discarded, $100 \mu \mathrm{L}$ of DMSO was added to each well, and the absorption was measured at $570 \mathrm{~nm}$.

\section{Determination of Silver Content in 2D Keratinocytes and EpiKutis Model}

Two hundred microliters of $500 \mu \mathrm{g} / \mathrm{mL}$ AgNPs were added to the surface of the $3 \mathrm{D}$ model, and $200 \mu \mathrm{L}$ of $14 \mu \mathrm{g} / \mathrm{mL}$ AgNPs were added to the 2D keratinocyte culture. The equivalent dose was $0.28 \mathrm{ng}$ per cell. Both test groups were cultured for $24 \mathrm{hrs}$ at $37^{\circ} \mathrm{C}$ in $5 \% \mathrm{CO}_{2}$. Then, the $3 \mathrm{D}$ model cells, the media above and below the model cells, and the 2D keratinocytes, were collected and digested with nitric acid for analysis of silver content using ICP-MS as previously described. ${ }^{32}$

\section{Assessment of Oxidative Stress and Inflammatory Mediators}

After 24 hrs of exposure to AgNPs, the media from the 3D model and 2D keratinocytes were collected for quantitation of IL-1 $\alpha$, IL-6, IL-8, and TNF- $\alpha$ using the corresponding ELISA kits according to the manufacturer's instructions. The 3D model preparations were transferred to aseptic EP tubes with
$300 \mu \mathrm{L}$ of saline, and the cells were homogenized. After centrifugation at 3,000 rpm for $10 \mathrm{mins}$, the supernatants were collected for analysis of SOD and MDA according to the kit manufacturer's instructions. The 2D keratinocyte cultures were placed on ice and rinsed twice with PBS. Three hundred microliters of precooled cell lysis solution (1\% Triton $\mathrm{X}-100$ and $1 \mathrm{mmol} \cdot \mathrm{L}^{-1} \mathrm{PMSF}$ in PBS) were added to the cells. After incubation for 5 mins, the cells were scraped using a cell scraper. The cell lysates were collected and transferred to $1.5-\mathrm{mL} \mathrm{EP}$ tubes, then incubated on ice for 20 mins accompanied by intermittent three times vortex oscillation. After centrifugation at $12,000 \mathrm{rpm}$ for $20 \mathrm{mins}$ at $4^{\circ} \mathrm{C}$, the supernatants were collected for quantitation of SOD and MDA according to the kit manufacturer's instructions.

For ROS Detection, the 3D model preparations and 2D keratinocytes were digested in $0.25 \%$ trypsin-EDTA to prepare single-cell suspensions. Reactive oxygen species were measured using flow cytometry (Bricyte E6, Mindray, China) according to the kit manufacturer's instructions.

\section{Transmission Electron Microscopy Analysis of AgNPs in 2D Keratinocytes and EpiKutis Model}

Transmission electron microscopy was performed as previously described. ${ }^{32}$ Following exposure to AgNPs, the EpiKutis model and 2D keratinocyte preparations were collected and immediately fixed in $2.5 \%$ glutaraldehyde overnight. After washing, the models were fixed using $1 \% \mathrm{OsO}_{4}$ for $1.5 \mathrm{hrs}$, dehydrated using a 50-100\% ethanol gradient, then transferred to a gradient of embedding solutions to prepare embedding blocks. Ultrathin sections from the embedding block were collected onto a copper grid for visualization using field-emission high-resolution TEM (JEM-2100F, JEOL, Japan).

\section{Statistical Analyses}

Differences between groups were determined using one-way analysis of variance (ANOVA). $\mathrm{P}<0.05$ was considered statistically significant. All datasets are presented as the mean \pm SD from at least three independent experiments.

\section{Results and Discussion Construction and Characterization of the EpiKutis Model}

The 3D epidermis model, EpiKutis, was developed in this study through the reconstruction of keratinocytes. Histological analysis showed that this model consisted of multiple layers of 
viable epithelial cells, including a basal layer, a stratum spinous layer, a stratum granular layer, and a stratum corneum layer (Figure 1A). This structure was very similar to that of the normal human epidermis. To identify the quality control criteria of EpiKutis ${ }^{\circledR}$ for batch release, cell viability and barrier function were evaluated according to OECD Guidelines for the Testing of Chemicals - In vitro Skin Irritation: Reconstructed Human Epidermis Test Method. ${ }^{33}$ Cell viability of the EpiKutis ${ }^{\circledR}$ model was assessed using the MTT assay. The range of optical density (OD) values between which the number of living cells could be calculated was 1.0-2.5. The results from 60 batches fell within this range, as shown in Figure 1B. The stratum corneum and its lipid composition are essential to the skin barrier function of reconstructed epidermis models. An appropriate model should be able to resist rapid penetration by cytotoxic chemicals, such as Triton $\mathrm{X}-100$, as estimated by $\mathrm{ET}_{50}$ values. ${ }^{34}$ The $\mathrm{ET}_{50}$ range of EpiKutis for resistance to penetration by $1 \%$ Triton X-100 was $1-3$ hrs. The results from 60 batches were within this range, as shown in Figure 1C. The quality control criteria for EpiKutis are summarized in Table 1.

Multiple human epidermal models have been established in recent years. EpiDerm ${ }^{\mathrm{TM}}$, EpiSkin ${ }^{\mathrm{TM}}$, and SkinEthic are the most widely used commercial epidermal models. ${ }^{33}$ They are generated by culturing differentiated keratinocytes on acellular or fibroblast-populated dermal substrates. ${ }^{35-41}$ Each of these epidermal models exhibit a multilayered epithelium and display characteristic epidermal ultrastructures. ${ }^{40,42,43}$ However, there are several differences among these epidermal models related to the number of living cell layers, the expression, and localization of cutinized envelope proteins, ${ }^{44}$ and differential cell viability and barrier function. Many different skin models have been successfully used to evaluate chemical-induced skin corrosion and irritation. ${ }^{45-48}$ However, few of these models have been used to evaluate the toxicity of AgNPs. To accurately evaluate toxicity of AgNPs, we developed the 3D EpiKutis model to provide a better simulation of in vivo conditions. Our approach was similar to that used to

\section{A}

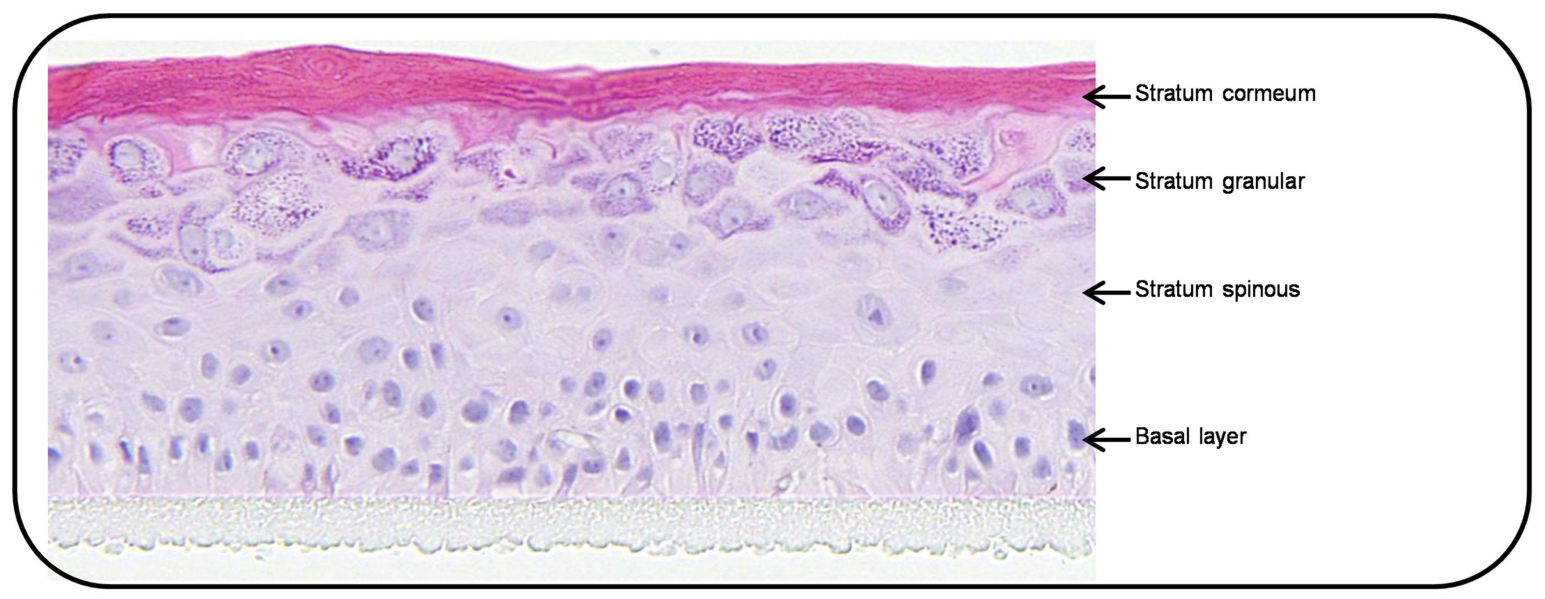

B

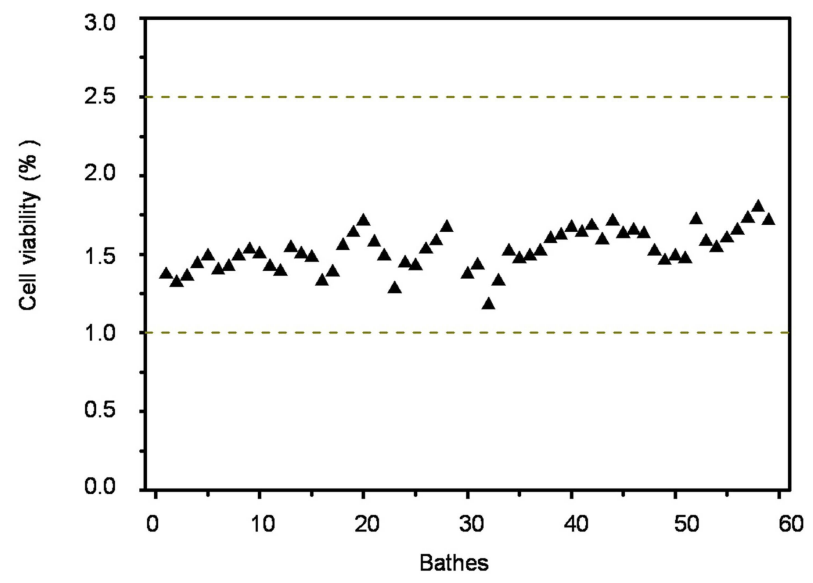

C

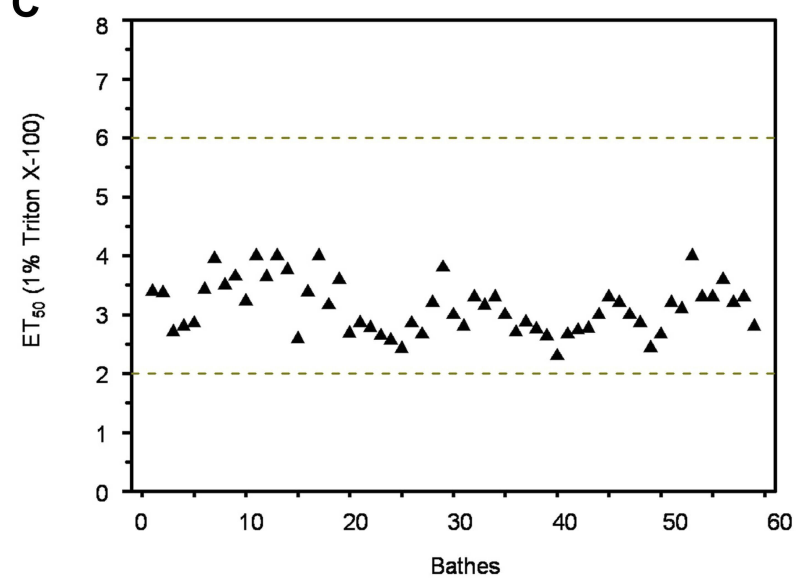

Figure I Characterization of 3D EpiKutis ${ }^{\circledR}$ model. Histological examination (A), viability (B) and ET50 (C) analysis of EpiKutis model. 
Table I The Quality Control Criteria of EpiKutis ${ }^{\circledR}$ Model

\begin{tabular}{|c|c|}
\hline Items & Criteria \\
\hline \multirow[t]{3}{*}{ Apparent evaluation } & Dry, flat, and no shrink. \\
\hline & Intact epidermis construction \\
\hline & I. Stratum basal layer; \\
\hline Histological examination & 2. Stratum spinosum layer; \\
\hline \multirow[t]{2}{*}{ (Hematoxylin and eosin stain) } & 3. Stratum granulosum layer; \\
\hline & 4. Multilayered stratum corneum. \\
\hline Cell viability & $1.0 \leq \mathrm{OD}_{570} \leq 2.5$ \\
\hline Barrier function ${ }^{\mathrm{a}}$ & $\mathrm{I}$ hrs $\leq \mathrm{ET}_{50} \leq 3 \mathrm{hrs}$ \\
\hline
\end{tabular}

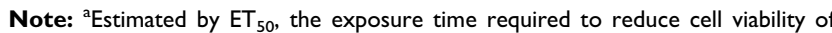
EpiKutis ${ }^{\circledR}$ by $50 \%$ when added $0.1 \%$ Triton- $X 100$.

produce Episkin ${ }^{\circledR}{ }^{48}$ EpiKutis was comprised of approximately 10 living cell layers. The protocols used to evaluate cell viability and barrier function in EpiKutis in response to AgNP exposure were similar to those used for evaluation of the EpiDerm ${ }^{\mathrm{TM}}$, EpiSkin ${ }^{\mathrm{TM}}$, and SkinEthic models. ${ }^{49-51}$ Our results showed that the EpiKutis model exhibited a normal epidermal-laminated structure and good barrier function, which suggested that the EpiKutis model was an appropriate 3D-reconstructed epidermal model, and was suitable for the assessment of AgNP toxicity.

\section{Physicochemical Properties of AgNPs}

Transmission electron microscopy imaging indicated that AgNPs were spherical and mono-dispersed (Figure 2A). The average diameter of the AgNPs was $9.1 \pm 2.6 \mathrm{~nm}$, as determined using ImageJ software. Dynamic light scattering (DLS) analysis showed that the hydrodynamic diameter of AgNPs was $14.0 \pm 1.6 \mathrm{~nm}$ (Figure 2B) with a zeta potential of $-27.2 \pm 1.0 \mathrm{mV}$. Analysis using UV-vis showed that AgNPs had a relatively narrow size distribution, and the $\lambda_{\max }$ was $420 \mathrm{~nm}$ (Figure 2C). X-ray photoelectron spectroscopy (XPS) analysis confirmed that silver was present in the nanoparticles (Figure 2D).

\section{Toxic Effects of AgNPs}

To assess skin toxicity of AgNPs, the reconstructed human epidermal model EpiKutis was used to mimic human skin. Assessment of toxicity using this model was compared to toxicity in 2D cell culture. Treatment of EpiKutis and keratinocytes for 24 hrs resulted in less cytotoxicity in EpiKutis than in 2D keratinocytes, as shown in Figure 3. The relative cell viability following exposure to AgNPs was greater than $80 \%$ in EpiKutis, even in response to the highest dose of AgNPs. In addition, the barrier function $\left(\mathrm{ET}_{50}\right)$ of the EpiKutis model was not significantly different than the value at the initial timepoint. In contrast, AgNPs induced cytotoxicity in a dosedependent manner in 2D keratinocytes, as evidenced by decreased cell viability from $63 \%$ to $11 \%$ with increasing doses of AgNPs (Figure 3A). Furthermore, cell membrane permeability, as determined by LDH release, increased from $8 \%$ to $16 \%$ with increasing doses of AgNPs (Figure 3B).

Our results showed that AgNPs induced significant changes in cell viability and membrane permeability in $2 \mathrm{D}$ keratinocytes. Reduced toxicity of AgNPs in the 3D model indicated that the $3 \mathrm{D}$ structure and the morphology of the model played a critical role in tissue viability and barrier function. A previous study evaluated acute exposure of tissue models and cells to silver nanowires (AgNW). The results of this study showed that there was no decrease in viability of human-reconstructed epidermis (RHE) after 20 mins or 42 hrs of contact with silver nanowires. ${ }^{52}$ These results indicated that an epidermal model with a stratum corneum and an extracellular matrix could protect keratinocytes from cytotoxicity resulting from direct exposure to silver ions or silver nanoparticles. Therefore, 3D epidermal models may be more suitable than 2D models for evaluation of skin exposure risk associated with nanomaterials.

\section{Assessment of AgNP Penetration}

To further investigate penetration and cellular uptake of AgNPs in the 3D EpiKutis model and in 2D keratinocytes, silver (Ag) content was quantified using ICP-MS after $24 \mathrm{hrs}$ of exposure to AgNPs. The initial total AgNP doses were 100 $\mu \mathrm{g}$ in the EpiKutis model and $2.8 \mu \mathrm{g}$ in $2 \mathrm{D}$ keratinocytes, with an equivalent dose of $0.28 \mathrm{ng}$ per cell. As shown in Figure 4, the penetration percentages of $\mathrm{Ag}$ in the EpiKutis model and in keratinocytes were $0.9 \pm 0.1 \%$ and $8.8 \pm 0.6 \%$, respectively. In the EpiKutis model, $76.7 \pm 7.4 \mu \mathrm{g}$ of silver was detected in the medium in the upper chamber, but only $0.9 \pm 0.1 \mu \mathrm{g}$ was detected within the tissue, which suggested that the 3D EpiKutis model protected cells from invasion by extrinsic nanoparticles. These results correlated well with the toxicity differences observed between the 3D EpiKutis model and 2D keratinocytes.

We then investigated the distribution of AgNPs in the 2D keratinocytes and EpiKutis model. However, AgNPs were not found in the 3D EpiKutis model (Figure 5A-D). But the penetration and distribution of AgNPs in 2D keratinocytes were confirmed by the TEM-EDS analysis (Figure 5E-G). This result may have been due to poor penetration of intact 
A

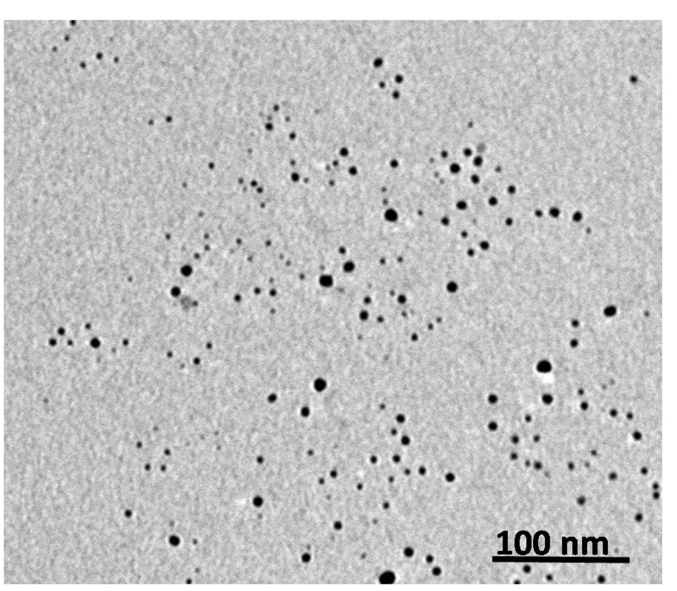

C

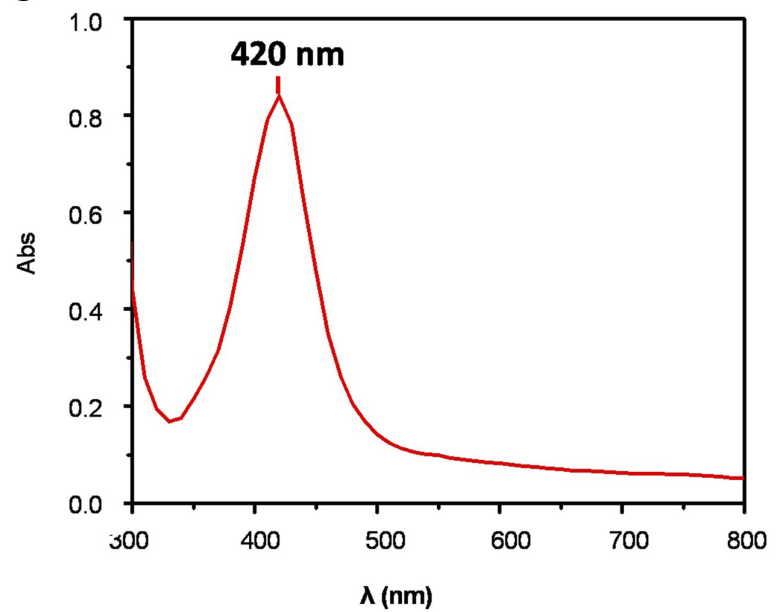

B

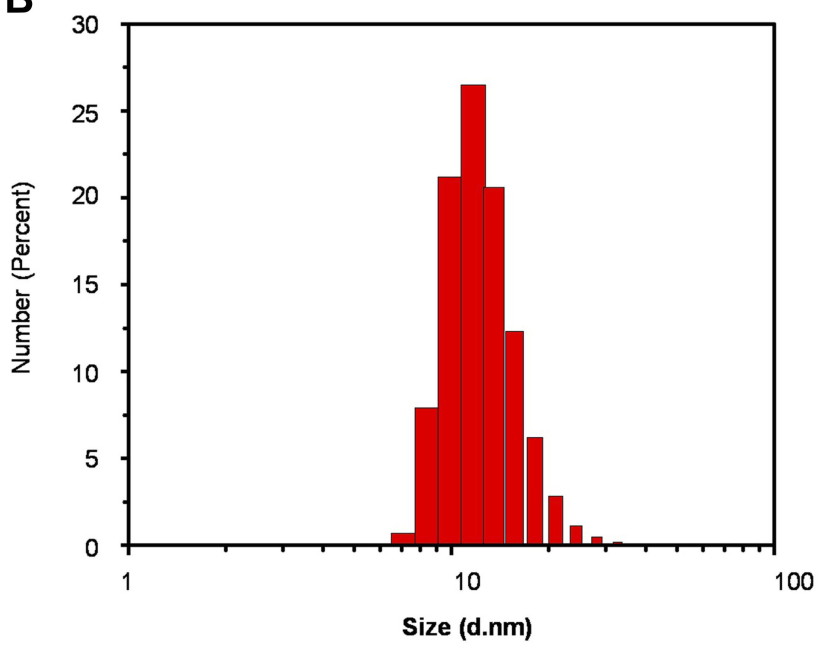

D

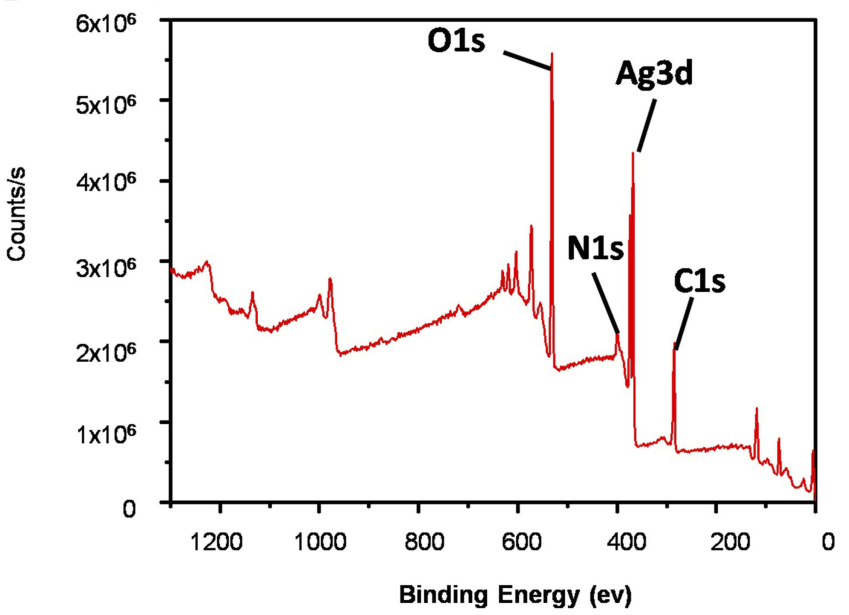

Figure 2 Physicochemical characterization of AgNPs. (A) The representative TEM image.

(B-D) DLS, UV and XPS analysis of AgNPs, respectively.

particles into the EpiKutis model, which further supported that EpiKutis exhibited good barrier function.

Intact epidermis exhibits an effective barrier function. Larese et al suggested that increased dermal penetration of $25 \mathrm{~nm}$ AgNPs could occur in damaged human skin. ${ }^{53}$ The barrier function of the epidermis has been reported for many nanomaterials, including carbon-based nanoparticles, titanium dioxide, zinc oxide, and gold nanoparticles. ${ }^{1}$ A study by Sylvia G. Lehmann showed that AgNW did not penetrate into a 3D RHE, but 2D primary keratinocytes extensively internalized AgNW. ${ }^{52}$ These results were consistent with our findings that 3D EpiKutis exhibited good barrier function, resulting in protection against AgNP-induced damage.

\section{AgNP-Induced Oxidative Stress}

The relative amounts of ROS, MDA, and SOD were measured to evaluate AgNP-induced oxidative stress.
No significant changes in intracellular ROS, MDA, or SOD were observed in the EpiKutis models in response to treatment with $0.28 \mathrm{ng}$ per cell AgNPs (Figure 6). However, the levels of ROS and MDA were significantly increased in 2D keratinocytes exposed to an equivalent dose of AgNPs compared to those in the control group (Figure 6A and B). In addition, levels of the phase II antioxidant enzyme SOD were significantly decreased in 2D keratinocytes (Figure 6C). Inclusion of $10 \mathrm{mM}$ NAC, an antioxidant, in the 2D keratinocytes cell culture medium blocked AgNP-induced increases in ROS and MDA and prevented AgNP-induced decreases in SOD, which indicated that oxidative damage may be an effector of AgNP-related toxicity. These results showed that AgNPs did not induce oxidative stress in the 3D EpiKutis model, but did induce oxidative stress in $2 \mathrm{D}$ keratinocytes. 

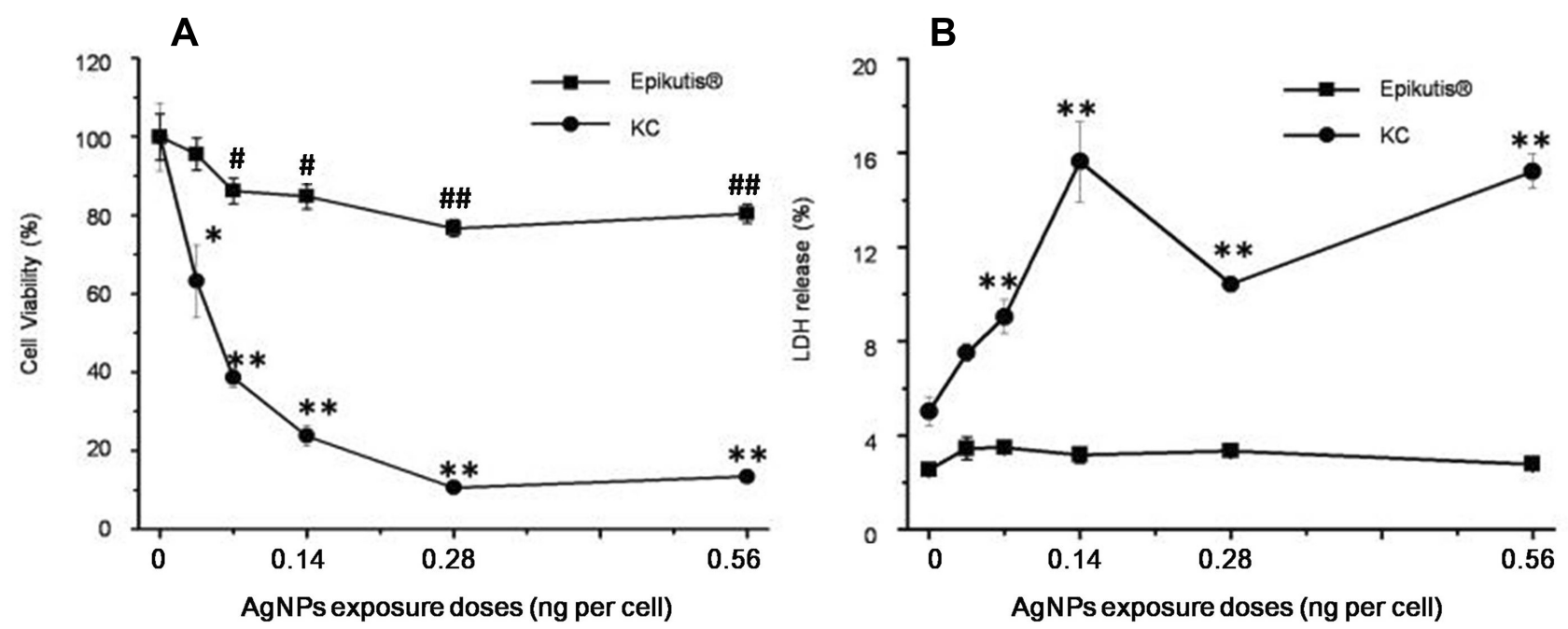

Figure 3 Toxic effects of AgNPs. Viability $(\mathbf{A})$ and $\mathrm{LDH}$ content $(\mathbf{B})$ of $3 \mathrm{D}$ EpiKutis ${ }^{\circledR}$ and $2 \mathrm{D} \mathrm{KC}$ cells after treatment with $\mathrm{AgNPs}$ for 24 hrs at the equivalent concentration, as determined by MTT and LDH assays. (Mean \pm SD, $n=3 ;{ }^{*}<0.05$ and ${ }^{* *} p<0.01$ indicating the statistical difference between the groups of $2 \mathrm{D} K C$ cell; \#p<0.05 and \#\#p<0.01 indicating the statistical difference between the groups of EpiKutis $\left.{ }^{\circledR}\right)$.
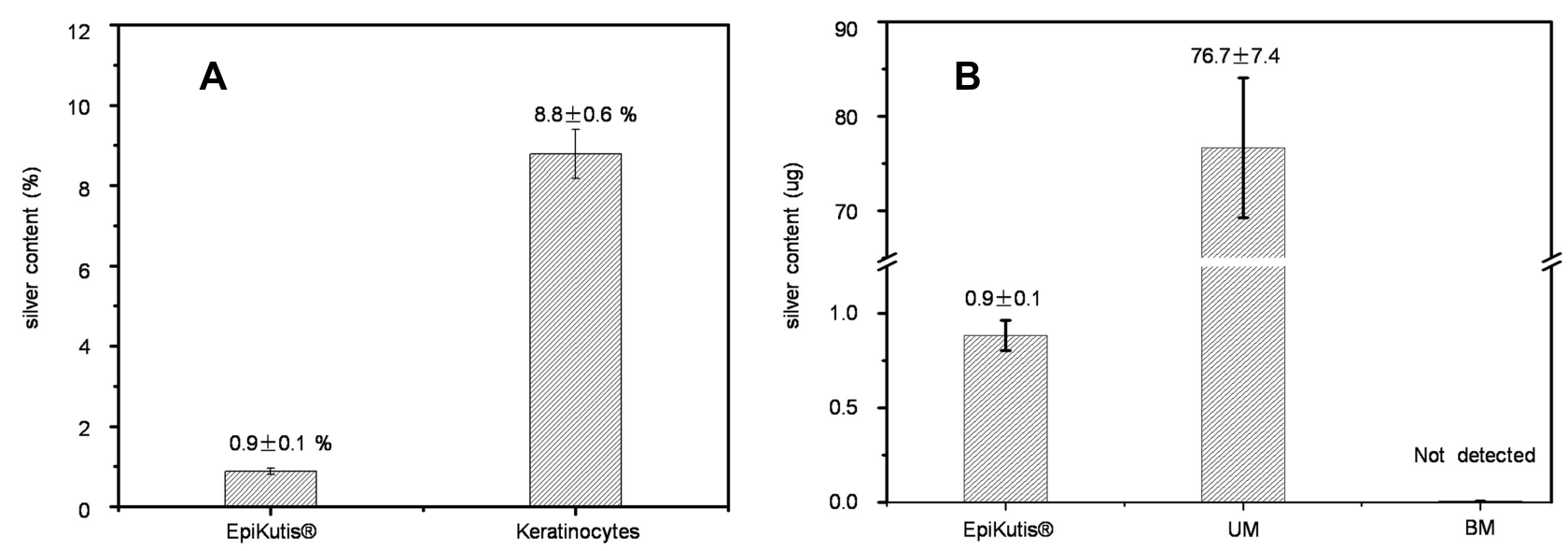

Figure 4 Silver content in the EpiKutis ${ }^{\circledR}$ model and 2D Keratinocytes after AgNPs exposure for 24 hrs at the equivalent dose of 0.28 ng per cell. (A) Silver content in EpiKutis model and 2D Keratinocytes. (B) Distribution of silver in the EpiKutis model (Mean $\pm S D, n=3$ ).

Abbreviations: UM, upper medium; BM, bottom medium.

Oxidative stress reflects an imbalance between ROS and reactive intermediates responsible for protection against ROS. ${ }^{54}$ According to the hierarchical oxidative stress model, lower levels of oxidative stress are associated with increased expression of antioxidant and detoxification enzymes, such as SOD, a phase II antioxidant enzyme. Disruption of this antioxidant response may result in negative health effects in response to exposure to external materials. Higher levels of oxidative stress result in inflammation and cytotoxicity, which can lead to apoptosis or necrosis. ${ }^{55-57}$ Previous studies have shown that oxidative stress may be an early event in toxicity induced by nanoparticles. ${ }^{55}$ Data from in vitro and in vivo studies indicated that oxidative stress may play an important role AgNP-induced toxicity. ${ }^{58-62}$ In the present study, AgNPs were cytotoxic toward 2D keratinocytes. However, treatment with up to $0.28 \mathrm{ng}$ of AgNPs per cell did not induce oxidative damage in the EpiKutis model, potentially due to excellent barrier function, complex cell-cell interactions, and the resulting gradient of oxygen and nanoparticle concentrations in the 3D environment. ${ }^{21}$ It was reported that oxidation of AgNPs in a complex microenvironment was a slow process, and the balance of autonomous regulating on oxidative stress state in cells could maintain for 

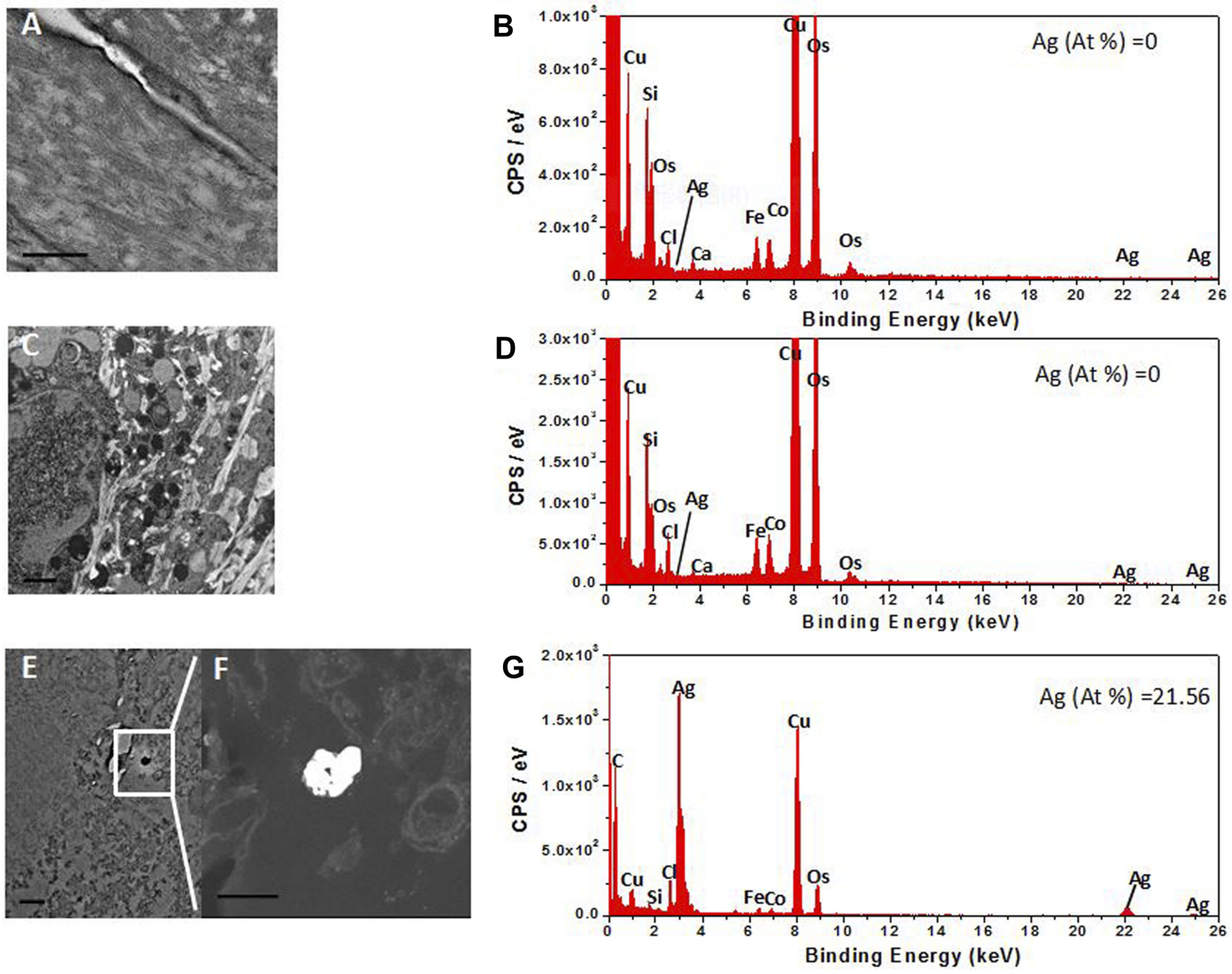

Figure 5 TEM imaging and EDS elemental graph of AgNPs. Figures $(\mathbf{A}, \mathbf{C})$ were the TEM images of the stratum corneum layer and cell layer of $3 D$ EpiKutis ${ }^{\circledR}$ respectively, of which EDS energy peaks of the detected elements were shown in the figure (B, D) correspondingly. Figure (E) was the TEM image of 2D KC cells, of which EDS energy peaks of the detected elements were shown in the figure (G). Figure (F) was the magnified TEM dark-field image of the object in the square frame of figure (E). The 3D EpiKutis and 2D KC cells were exposed to the equivalent doses of $0.28 \mathrm{ng}$ AgNPs per cell. The scale bar in the figure (F) was $200 \mathrm{~nm}$, the scale bar in other figures was $500 \mathrm{~nm}$.

$24 \mathrm{hrs}$ in the experiment. ${ }^{62}$ According to previous studies, chronic intracellular ROS accumulation could overwhelm the cellular antioxidant defense system, resulting in increased AgNP-induced oxidative stress over a $48 \mathrm{hrs}$ time period. ${ }^{5,63,64}$

\section{AgNP-Induced Release of Pro-Inflammatory Cytokines}

To evaluate AgNP-induced inflammation, we quantified the pro-inflammatory cytokines IL- $1 \alpha$, IL-6, IL- 8 , and TNF- $\alpha$. As shown in Figure 7A, increased secretion of proinflammatory cytokines (IL-1 $\alpha$, IL-6, and IL-8) was observed in 2D keratinocyte cultures following exposure to $0.28 \mathrm{ng}$ of AgNPs per cell for $24 \mathrm{hrs}$. However, AgNPs did not induce increased cytokine secretion in the $3 \mathrm{D}$ EpiKutis model
(Figure 7B). These results agreed with our findings that AgNPs induced cytotoxicity in 2D cultures, but not in the 3D EpiKutis model.

Inflammation is an early immunological response to exogenous materials. Keratinocytes can produce proinflammatory cytokines, such as IL-8, IL-6, TNF- $\alpha$, and IL-1 $\beta$, which play crucial roles in the inflammatory and immunologic reactions of skin tissue to irritants. ${ }^{65-68}$ In vitro exposure of immune cells ${ }^{69-71}$ and epithelial cells, ${ }^{63,72,73}$ and in vivo exposure, to AgNPs can induce the release of pro-inflammatory cytokines. ${ }^{74}$ For example, AgNPs with diameters of $15 \mathrm{~nm}, 30 \mathrm{~nm}$, and $55 \mathrm{~nm}$ induced secretion of pro-inflammatory cytokines such as TNF- $\alpha$, IL-1 $\beta$, and macrophage inflammatory protein-2 (MIP-2) from alveolar macrophages. ${ }^{60}$ Meghan et al 

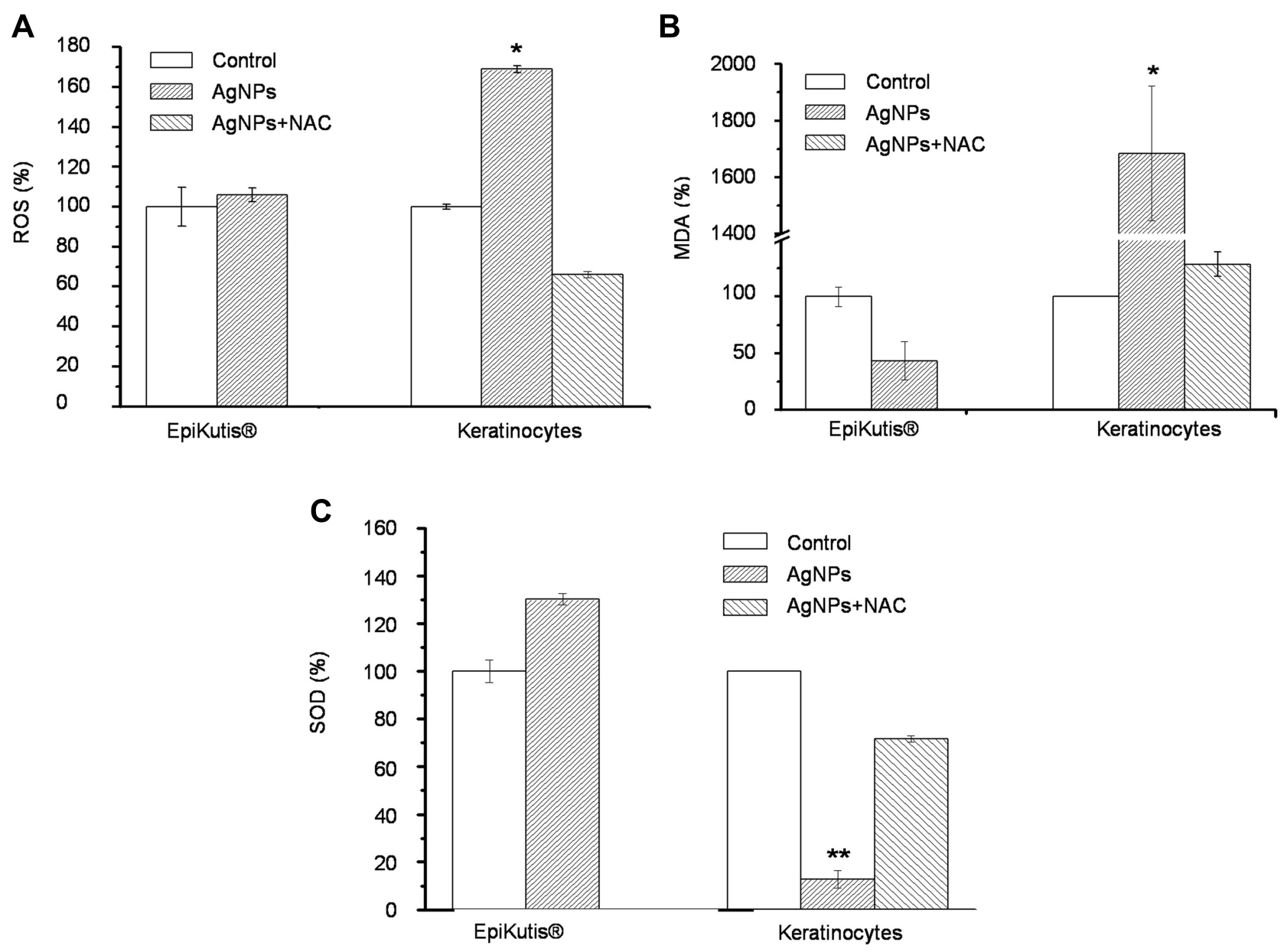

Figure 6 Oxidative stress analysis of EpiKutis ${ }^{\circledR}$ and KC cells. The relative level of ROS (A), MDA (B) and SOD (C) in 3D EpiKutis model and 2D KC cells after the treatment with AgNPs for $24 \mathrm{hrs}$ at the equivalent dose of $0.28 \mathrm{ng}$ AgNPs per cell. Ten millimolar N-acetylcysteine (NAC) was used as an antioxidant against oxidative damages (Mean $\pm S D, n=3, * p<0.05, * * p<0.01$ ).

A

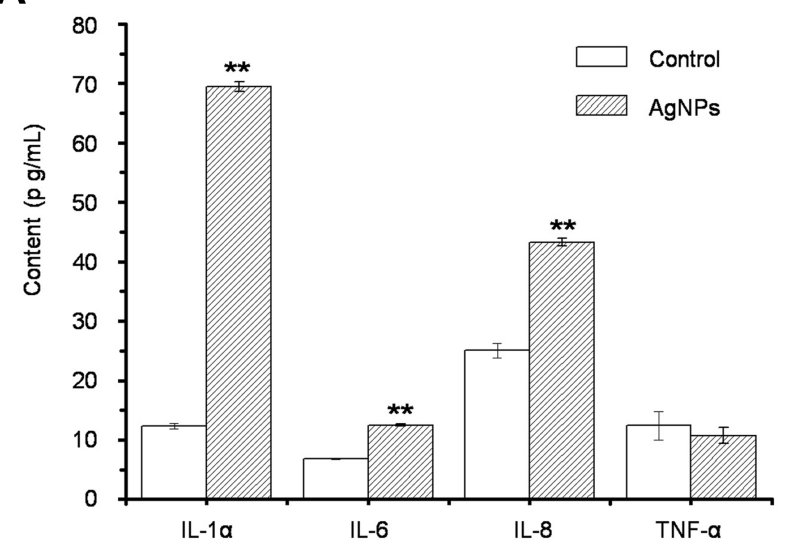

B

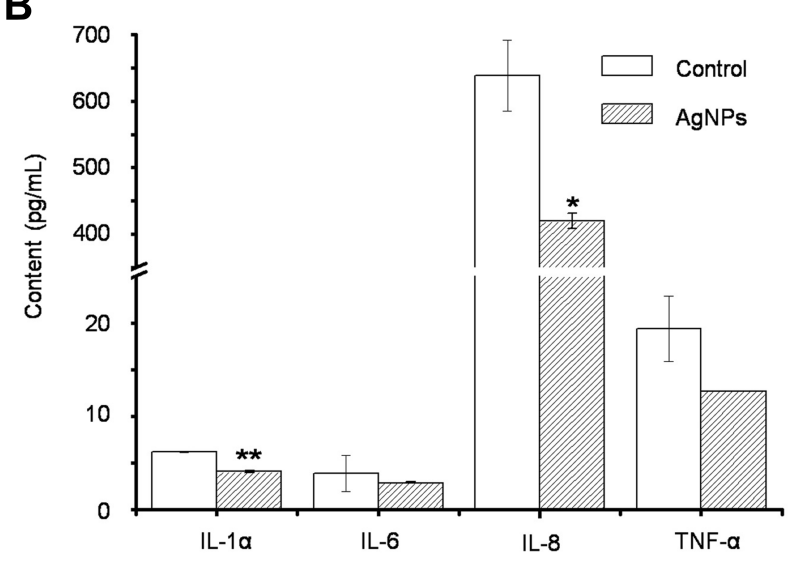

Figure 7 The release of inflammatory factors in 2D KC cells $(\mathbf{A})$ and $3 \mathrm{D}$ EpiKutis ${ }^{\circledR}$ model $(\mathbf{B})$ after the treatment with $\mathrm{AgNPs}$ for 24 hrs at the equivalent dose of $0.28 \mathrm{ng}$ AgNPs per cell (Mean $\pm S D, n=3,{ }^{*} p<0.05$, ${ }^{* *} p<0.0$ I).

compared AgNP-induced skin toxicity in porcine skin (in vivo) and keratinocytes (in vitro), and found that AgNPs induced a dose-dependent decrease in keratinocyte viability, and dose-dependent increases in IL-1 $\beta$, IL-6, IL-8, and TNF- $\alpha$ levels after $24 \mathrm{hrs}$ of exposure. In contrast, AgNPs only induced focal inflammation in skin after 
14 days of administration. ${ }^{30}$ These findings agreed with our results showing that AgNPs induced increased cytokine secretion from 2D keratinocytes, but not from $3 \mathrm{D}$ EpiKutis.

As shown in Figure 7B, levels of inflammatory cytokines, particularly IL- $1 \alpha$ and IL-8, were decreased following exposure of 3D EpiKutis to AgNPs. These results indicated that keratinocytes in the EpiKutis model were protected from direct exposure to AgNPs, as evidenced by poor penetration of $\mathrm{Ag}$ into the EpiKutis tissue $(0.9 \pm 0.1 \%)$, and by unchanged tissue viability and LDH release. Therefore, the cells in the EpiKutis model maintained a balance between inflammatory and anti-inflammatory responses through selfregulation, resulting in protection against mild AgNPinduced oxidative stress. This balance resulted in reduced inflammation in EpiKutis compared to that in 2D keratinocytes, as evidenced by reduced secretion of inflammatory cytokines. However, more intense stimulation could disrupt the balance between the cellular inflammatory response and the cellular anti-inflammatory response, which was observed in $2 \mathrm{D}$ keratinocyte cultures, as evidenced by increased levels of pro-inflammatory cytokines and decreased cell viability.

Cells within the EpiKutis model were protected from AgNPs due to good barrier function, which was consistent with findings that only minimal silver penetrated the skin in vivo. ${ }^{75}$ The results of this study demonstrated that the evaluation of nanoparticles should be performed using $3 \mathrm{D}$ epidermal models to provide a better estimate of in vivo effects.

\section{Conclusion}

In this study, the reconstructed 3D epidermal model EpiKutis showed good tissue viability (OD values between 1.0 and 2.5) and good barrier function $\left(\mathrm{ET}_{50}\right.$ between 1 and $3 \mathrm{~h}$ ). Therefore, this model was used to evaluate AgNP toxicity. In vitro evaluation of EpiKutis and 2D keratinocytes showed that equivalent doses of AgNPs resulted in significant oxidative damage- and inflammation-related cytotoxicity in 2D keratinocyte cultures, but not in 3D EpiKutis preparations. The barrier function in the EpiKutis model was evidenced by reduced silver permeation and penetration. Therefore, the present study suggested that skin toxicity studies using the $3 \mathrm{D}$ EpiKutis model might be more likely to reflect real physiological responses to AgNPs than 2D monolayer models. Our study showed that the EpiKutis model may be appropriate for the evaluation of nanotoxicity, particularly with regard to AgNPs. Furthermore, use of this model should be considered for the evaluation of medical products embedded with nanomaterials.

\section{Acknowledgments}

This study is financially supported by the Ministry of Science and Technology of China (2016YFA0201600, 2016YFA02 00903); the National Natural Science Foundation of China (grant number 31600814).

\section{Disclosure}

There is no conflict of interest to declare in this study.

\section{References}

1. Crosera M, Bovenzi M, Maina G, et al. Nanoparticle dermal absorption and toxicity: a review of the literature. Int Arch Occup Environ Health. 2009;82(9):1043-1055. doi:10.1007/s00420-009-0458-x

2. Augustine R, Kalarikkal N, Thomas S. Electrospun PCL membranes incorporated with biosynthesized silver nanoparticles as antibacterial wound dressings. Appl Nanosci. 2016;6(3):337-344. doi:10.1007/ s13204-015-0439-1

3. Augustine R, Augustine A, Kalarikkal N, et al. Fabrication and characterization of biosilver nanoparticles loaded calcium pectinate nano-micro dual-porous antibacterial wound dressings. Prog Biomater. 2016;5(3-4):223-235. doi:10.1007/s40204-016-0060-8

4. Wijnhoven S, Peijnenburg W, Herberts C, et al. Nano-silver a review of available data and knowledge gaps in human and environmental risk assessment. Nanotoxicology. 2009;3(2):109-138. doi:10.1080/17435390902725914

5. AshaRani PV, Low Kah Mun G, Hande MP, et al. Cytotoxicity and genotoxicity of silver nanoparticles in human cells. ACS Nano. 2009;3(2):279-290. doi:10.1021/nn800596w

6. Shahbazzadeh D, Ahari H, Motalebi AA, et al. In vitro effect of Nanosilver toxicity on fibroblast and mesenchymal stem cell lines. Iran J Fish Sci. 2011;10(3):487-496. doi:10.1093/icesjms/ fsr023

7. Kim S, Choi JE, Choi J, et al. Oxidative stress-dependent toxicity of silver nanoparticles in human hepatoma cells. Toxicol in Vitro. 2009;23(6):1076-1084. doi:10.1016/j.tiv.2009.06.001

8. Antony JJ, Sivalingam P, Chen B. Toxicological effects of silver nanoparticles. Environ Toxicol Pharmacol. 2015;40(3):729-732. doi:10.1016/j.etap.2015.09.003

9. Jones CF, Grainger DW. In vitro assessments of nanomaterial toxicity. Adv Drug Deliv Rev. 2009;61(6):438-456. doi:10.1016/j. addr.2009.03.005

10. Nel A, Xia T, Meng H, et al. Nanomaterial toxicity testing in the 21st century: use of a predictive toxicological approach and high-throughput screening. Acc Chem Res. 2013;46(3):607-621. doi:10.1021/ar300022h

11. Johnston HJ, Hutchison G, Christensen FM, et al. A review of the in vivo and in vitro toxicity of silver and gold particulates: particle attributes and biological mechanisms responsible for the observed toxicity. Crit Rev Toxicol. 2010;40(4):328-346. doi:10.3109/ 10408440903453074

12. Lee J, Cuddihy MJ, Kotov NA. Three-dimensional cell culture matrices: state of the art. Tissue Eng Part B Rev. 2008;14(1):61-86. doi:10.1089/teb.2007.0150

13. Sayes CM, Reed KL, Warheit DB. Assessing toxicity of fine and nanoparticles: comparing in vitro measurements to in vivo pulmonary toxicity profiles. Toxicol Sci. 2007;97(1):163-180. doi:10.1093/ toxsci $/ \mathrm{kfm} 018$ 
14. Astashkina A, Mann B, Grainger DW. A critical evaluation of in vitro cell culture models for high-throughput drug screening and toxicity. Pharmacol Ther. 2012;134(1):82-106. doi:10.1016/j.pharmthera.2012. 01.001

15. Dhawan A, Sharma V. Toxicity assessment of nanomaterials: methods and challenges. Anal Bioanal Chem. 2010;398(2):589-605. doi:10.1007/s00216-010-3996-x

16. Fröhlich E. Comparison of conventional and advanced in vitro models in the toxicity testing of nanoparticles. Artif Cells Nanomed Biotechnol. 2018;46(sup2):1091-1107. doi:10.1080/21691401.2018.1479709

17. Danilenko DM, Phillips GD, Diaz D. In vitro skin models and their predictability in defining normal and disease biology, pharmacology, and toxicity. Toxicol Pathol. 2016;44(4):555-563. doi:10.1177/ 0192623316632074

18. Huh D, Hamilton GA, Ingber DE. From 3D cell culture to organs-onchips. Trends Cell Biol. 2011;21(12):745-754. doi:10.1016/j. tcb.2011.09.005

19. Joris F, Manshian BB, Peynshaert K, et al. Assessing nanoparticle toxicity in cell-based assays: influence of cell culture parameters and optimized models for bridging the in vitro-in vivo gap. Chem Soc Rev. 2013;42(21):8339-8359. doi:10.1039/c3cs60145e

20. Yamada KM, Cukierman E. Modeling tissue morphogenesis and cancer in 3D. Cell. 2007;130(4):601-610. doi:10.1016/j.cell.2007.08.006

21. Drewitz M, Helbling M, Fried N, et al. Towards automated production and drug sensitivity testing using scaffold-free spherical tumor microtissues. Biotechnol J. 2011;6(12):1488-1496. doi:10.1002/ biot. 201100290

22. Mueller D, Krämer L, Hoffmann E, et al. 3D organotypic HepaRG cultures as in vitro model for acute and repeated dose toxicity studies. Toxicol in Vitro. 2014;28(1):104-112. doi:10.1016/j.tiv.2013.06.024

23. Lee J, Lilly GD, Doty RC, et al. In vitro toxicity testing of nanoparticles in 3D cell culture. Small. 2009;5(10):1213-1221. doi:10.1002/ smll.200801788

24. Huang K, Ma H, Liu J, et al. Size-dependent localization and penetration of ultrasmall gold nanoparticles in cancer cells, multicellular spheroids, and tumors in vivo. ACS Nano. 2012;6(5):4483-4493. doi: $10.1021 / \mathrm{nn} 301282 \mathrm{~m}$

25. Luo Y, Wang C, Hossain M, et al. Three-dimensional microtissue assay for high-throughput cytotoxicity of nanoparticles. Anal Chem. 2012;84(15):6731-6738. doi:10.1021/ac301191j

26. Movia D, Prina-Mello A, Bazou D, et al. Screening the cytotoxicity of single-walled carbon nanotubes using novel 3D tissue-mimetic models. ACS Nano. 2011;5(11):9278-9290. doi:10.1021/nn203659m

27. Wills JW, Hondow N, Thomas AD, et al. Genetic toxicity assessment of engineered nanoparticles using a 3D in vitro skin model (EpiDerm ${ }^{\mathrm{TM}}$ ). Part Fibre Toxicol. 2016;13(1):50. doi:10.1186/ s12989-016-0161-5

28. Marambio-Jones C, Hoek E. A review of the antibacterial effects of silver nanomaterials and potential implications for human health and the environment. J Nanopart Res. 2010;12(5):1531-1551. doi:10. 1007/s11051-010-9900-y

29. Prabha RD, Kandasamy R, Sivaraman US, et al. Antibacterial nanosilver coated orthodontic bands with potential implications in dentistry. Indian J Med Res. 2016;144(4):580-586. doi:10.4103/ 0971-5916.200895

30. Samberg ME, Oldenburg SJ, Monteiro-Riviere NA. Evaluation of silver nanoparticle toxicity in skin in vivo and keratinocytes in vitro. Environ Health Perspect. 2010;118(3):407-413. doi:10.1289/ ehp.0901398

31. Hyun JS, Lee BS, Ryu HY, et al. Effects of repeated silver nanoparticles exposure on the histological structure and mucins of nasal respiratory mucosa in rats. Toxicol Lett. 2008;182(1-3):24-28. doi:10.1016/j.toxlet.2008.08.003

32. Wu M, Guo H, Liu L, et al. Size-dependent cellular uptake and localization profiles of silver nanoparticles. Int $J$ Nanomedicine. 2019;14:4247-4259. doi:10.2147/IJN.S201107
33. Organization for Economic Cooperation and Development (OECD). Guidelines for the Testing of Chemicals. In vitro Skin Irritation: Reconstructed Human Epidermis Test Method. 2015.

34. Ponec M, Weerheim A, Kempenaar J, et al. Lipid composition of cultured human keratinocytes in relation to their differentiation. J Lipid Res. 1988;29(7):949-961. doi:10.1080/03003930.2014.930025

35. Pruniéras M, Régnier M, Woodley D. Methods for cultivation of keratinocytes with an air-liquid interface. $J$ Invest Dermatol. 1983;81(1 Suppl):28s-33s. doi:10.1111/1523-1747.ep12540324

36. Bell E, Ehrlich HP, Buttle DJ, et al. Living tissue formed in vitro and accepted as skin-equivalent tissue of full thickness. Science. 1981;211 (4486):1052-1054. doi:10.1126/science.7008197

37. Tinois E, Tiollier $J$, Gaucherand $M$, et al. In vitro and post-transplantation differentiation of human keratinocytes grown on the human type IV collagen film of a bilayered dermal substitute. Exp Cell Res. 1991;193(2):310-9.10. doi:10.16/00144827(91)90102-Z

38. Cannon CL, Neal PJ, Southee JA, et al. New epidermal model for dermal irritancy testing. Toxicol in vitro. 1994;8(4):889-891. doi:10.1016/0887-2333(94)90095-7

39. Rosdy M, Clauss LC. Terminal epidermal differentiation of human keratinocytes grown in chemically defined medium on inert filter substrates at the air-liquid interface. J Invest Dermatol. 1990;95 (4):409-414. doi:10.1111/1523-1747.ep12555510

40. Boyce S, Michel S, Reichert U, et al. Reconstructed skin from cultured human keratinocytes and fibroblasts on a collagen-glycosaminoglycan biopolymer substrate. Skin Pharmacol. 1990;3(2):136-143. doi:10.1159/000210860

41. Gibbs S, Vicanová J, Bouwstra J, et al. Culture of reconstructed epidermis in a defined medium at 33 degrees $\mathrm{C}$ shows a delayed epidermal maturation, prolonged lifespan and improved stratum corneum. Arch Dermatol Res. 1997;289(10):585-595. doi:10.1007/ s004030050244

42. Ponec M, Weerheim A, Kempenaar J, et al. The formation of competent barrier lipids in reconstructed human epidermis requires the presence of vitamin C. J Invest Dermatol. 1997;109(3):348-355. doi:10.1111/1523-1747.ep12336024

43. Boelsma E, Gibbs S, Faller C, et al. Characterization and comparison of reconstructed skin models: morphological and immunohistochemical evaluation. Acta Derm Venereol. 2000;80(2):82-88. doi:10.1080/ 000155500300013043

44. Netzlaff F, Lehr CM, Wertz PW, et al. The human epidermis models EpiSkin, SkinEthic and EpiDerm: an evaluation of morphology and their suitability for testing phototoxicity, irritancy, corrosivity, and substance transport. Eur J Pharm Biopharm. 2005;60(2):167-178. doi:10.1016/j.ejpb.2005.03.004

45. Chomiczewska D, Kieć-Swierczyńska M, Krecisz B. Irritant contact dermatitis. Part II. Evaluation evaluation of skin irritation potential of chemicals. Med Pr. 2009;60(3):209-214. doi:10.1111/j.15481387.2009.01074.x

46. El Ghalbzouri A, Siamari R, Willemze R, et al. Leiden reconstructed human epidermal model as a tool for the evaluation of the skin corrosion and irritation potential according to the ECVAM guidelines. Toxicol in Vitro. 2008;22(5):1311-1320. doi:10.1016/j. tiv.2008.03.012

47. Murthy PB, Kishore AS, Surekha P. Assessment of in vitro skin irritation potential of nanoparticles: RHE model. Methods Mol Biol. 2012;926:219-234. doi:10.1007/978-1-62703-002-1_16

48. Portes P, Grandidier MH, Cohen C, et al. Refinement of the Episkin protocol for the assessment of acute skin irritation of chemicals: follow-up to the ECVAM prevalidation study. Toxicol in vitro. 2002;16(6):765-770. doi:10.1016/S0887-2333(02)00090-5

49. Kandárová H, Liebsch M, Gerner I, et al. The EpiDerm test protocol for the upcoming ECVAM validation study on in vitro skin irritation tests-an assessment of the performance of the optimised test. Altern Lab Anim. 2005;33(4):351-367. doi:10.1177/026119290503300408 
50. Cotovio J, Grandidier MH, Portes P, et al. The in vitro skin irritation of chemicals: optimisation of the EPISKIN prediction model within the framework of the ECVAM validation process. Altern Lab Anim. 2005;33(4):329-349. doi:10.1016/S0960-894X(01)00566-2

51. Kandárová H, Liebsch M, Schmidt E, et al. Assessment of the skin irritation potential of chemicals by using the SkinEthic reconstructed human epidermal model and the common skin irritation protocol evaluated in the ECVAM skin irritation validation study. Altern Lab Anim. 2006;34(4):393-406. doi:10.1016/S1355-0306(05)71625-7

52. Lehmann SG, Gilbert B, Maffeis TG, et al. In vitro dermal safety assessment of silver nanowires after acute exposure: tissue vs. cell models. Nanomaterials. 2018;8(4). doi:10.3390/nano8040232

53. Larese FF, D'Agostin F, Crosera M, et al. Human skin penetration of silver nanoparticles through intact and damaged skin. Toxicology. 2009;255(1-2):33-37. doi:10.1016/j.tox.2008.09.025

54. Madl AK, Plummer LE, Carosino C, et al. Nanoparticles, lung injury, and the role of oxidant stress. Annu Rev Physiol. 2014;76:447-465. doi:10.1146/annurev-physiol-030212-183735

55. Nel A, Xia T, Mädler L, et al. Toxic potential of materials at the nanolevel. Science. 2006;311(5761):622-627. doi:10.1126/science. 1114397

56. Xiao GG, Wang M, Li N, et al. Use of proteomics to demonstrate a hierarchical oxidative stress response to diesel exhaust particle chemicals in a macrophage cell line. J Biol Chem. 2003;278 (50):50781-50790. doi:10.1074/jbc.M306423200

57. Ayres JG, Borm P, Cassee FR, et al. Evaluating the toxicity of airborne particulate matter and nanoparticles by measuring oxidative stress potential-a workshop report and consensus statement. Inhal Toxicol. 2008;20(1):75-99. doi:10.1080/08958370701665517

58. Tiwari DK, Jin T, Behari J. Dose-dependent in-vivo toxicity assessment of silver nanoparticle in Wistar rats. Toxicol Mech Methods. 2011;21(1):13-24. doi:10.3109/15376516.2010.529184

59. Chairuangkitti P, Lawanprasert S, Roytrakul S, et al. Silver nanoparticles induce toxicity in A549 cells via ROS-dependent and ROS-independent pathways. Toxicol in vitro. 2013;27(1):330-338. doi:10.1016/j.tiv.2012.08.021

60. Carlson C, Hussain SM, Schrand AM, et al. Unique cellular interaction of silver nanoparticles: size-dependent generation of reactive oxygen species. J Phys Chem B. 2008;112(43):13608-13619. doi:10.1021/jp712087m

61. Dubey P, Matai I, Kumar SU, et al. Perturbation of cellular mechanistic system by silver nanoparticle toxicity: cytotoxic, genotoxic and epigenetic potentials. Adv Colloid Interface Sci. 2015;221:4-21. doi:10.1016/j.cis.2015.02.007

62. Herzog F, Loza K, Balog S, et al. Mimicking exposures to acute and lifetime concentrations of inhaled silver nanoparticles by two different in vitro approaches. Beilstein J Nanotechnol. 2014;5:1357-1370. doi:10.3762/bjnano.5.149
63. Foldbjerg R, Dang DA, Autrup H. Cytotoxicity and genotoxicity of silver nanoparticles in the human lung cancer cell line, A549. Arch Toxicol. 2011;85(7):743-750. doi:10.1007/s00204-010-0545-5

64. Kawata K, Osawa M, Okabe S. In vitro toxicity of silver nanoparticles at noncytotoxic doses to HepG2 human hepatoma cells. Environ Sci Technol. 2009;43(15):6046-6051. doi:10.1021/es900754q

65. Allen DG, Riviere JE, Monteiro-Riviere NA. Identification of early biomarkers of inflammation produced by keratinocytes exposed to jet fuels jet A, JP-8, and JP-8(100). J Biochem Mol Toxicol. 2000;14 (5):231-237. doi:10.1002/1099-0461(2000)14:5<231::aid-jbt1>3.0. $\mathrm{CO} ; 2-\mathrm{K}$

66. Allen DG, Riviere JE, Monteiro-Riviere NA. Cytokine induction as a measure of cutaneous toxicity in primary and immortalized porcine keratinocytes exposed to jet fuels, and their relationship to normal human epidermal keratinocytes. Toxicol Lett. 2001;119(3):209-217. doi:10.1016/S0378-4274(00)00316-7

67. Gröne A. Keratinocytes and cytokines. Vet Immunol Immunopathol. 2002;88(1-2):1-12. doi:10.1016/s0165-2427(02)00136-8

68. Monteiro-Riviere NA, Baynes RE, Riviere JE. Pyridostigmine bromide modulates topical irritant-induced cytokine release from human epidermal keratinocytes and isolated perfused porcine skin. Toxicology. 2003;183(1-3):15-28. doi:10.1016/S0300-483X(02) 00421-3

69. Park EJ, Yi J, Kim Y, et al. Silver nanoparticles induce cytotoxicity by a Trojan-horse type mechanism. Toxicol in Vitro. 2010;24 (3):872-878. doi:10.1016/j.tiv.2009.12.001

70. Foldbjerg R, Olesen P, Hougaard M, et al. PVP-coated silver nanoparticles and silver ions induce reactive oxygen species, apoptosis and necrosis in THP-1 monocytes. Toxicol Lett. 2009;190 (2):156-162. doi:10.1016/j.toxlet.2009.07.009

71. Shin SH, Ye MK, Kim HS, et al. The effects of nano-silver on the proliferation and cytokine expression by peripheral blood mononuclear cells. Int Immunopharmacol. 2007;7(13):1813-1818. doi:10. 1016/j.intimp.2007.08.025

72. Park S, Lee YK, Jung M, et al. Cellular toxicity of various inhalable metal nanoparticles on human alveolar epithelial cells. Inhal Toxicol. 2007;19(Suppl 1):59-65. doi:10.1080/08958370701493282

73. Asharani P, Sethu S, Lim HK, et al. Differential regulation of intracellular factors mediating cell cycle, DNA repair and inflammation following exposure to silver nanoparticles in human cells. Genome Integr. 2012;3(1):2. doi:10.1186/2041-9414-3-2

74. Park EJ, Bae E, Yi J, et al. Repeated-dose toxicity and inflammatory responses in mice by oral administration of silver nanoparticles. Environ Toxicol Pharmacol. 2010;30(2):162-168. doi:10.1016/j. etap.2010.05.004

75. Nadworny PL, Landry BK, Wang J, et al. Does nanocrystalline silver have a transferable effect? Wound Repair Regen. 2010;18 (2):254-265. doi:10.1111/j.1524-475X.2010.00579.x
International Journal of Nanomedicine

\section{Publish your work in this journal}

The International Journal of Nanomedicine is an international, peerreviewed journal focusing on the application of nanotechnology in diagnostics, therapeutics, and drug delivery systems throughout the biomedical field. This journal is indexed on PubMed Central, MedLine, CAS, SciSearch ${ }^{\mathbb{R}}$, Current Contents ${ }^{\mathbb{B}} /$ Clinical Medicine,
Journal Citation Reports/Science Edition, EMBase, Scopus and the Elsevier Bibliographic databases. The manuscript management system is completely online and includes a very quick and fair peer-review

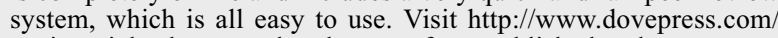
testimonials.php to read real quotes from published authors. 